\title{
Acknowledgement to Referees 2014
}

Received: 8 January 2015 / Accepted: 9 January 2015 /Published online: 4 February 2015

(C) Springer-Verlag Berlin Heidelberg 2015

The first 101 volumes of "The Science of Nature" were published under its former German title Naturwissenschaften. With volume 102, the former German title has been maintained as the subtitle to "The Science of Nature", recognising the journal's long-standing history (Thatje 2014, 2015). Here, I wish to thank all scientific peers who helped in assessing scholarly works submitted to the journal in 2014. All scholarly work published in the journal has been subjected to rigorous peer review. Peer review is a tedious and often a time consuming process but essential for maintaining and further developing scientific standards. In 2014 alone, 574 peers from 49 countries volunteered their time to support the journal and science, and many have assisted in more than one case, for which I am highly grateful. Average time from submission to first decisions was 22 days in 2014, which is exceptionally fast and the result of peers' commitment to the process. Peer review is not always an enjoyable process, neither for authors nor reviewers, but it does undoubtedly increase the quality of science and scientific publishing. On average, I seek the views of three independent referees, and in many cases, the journal's Associate Editors provide additional report on manuscripts. Often, referees are willing to again assess revised work, and this adds to the overall commitment to this process. Below is a list of the names of all colleagues who delivered a review in 2014, number of reviews in brackets if more than one. The information is derived from our online submission system; peers mostly provide the information themselves. Although this information should be correct, I would already like to apologise in case I may have missed recognising the name of any colleague involved in last year's peer-review process. Further, I would not like to miss the opportunity to thank my board of Associate Editors for their professional support in the assessment of hundreds of manuscripts we receive each year.

Sven Thatje

(Editor-in-Chief)

\section{References}

Thatje S (2014) Moving forward: change of journal title and continuous article publishing. Naturwissenschaften 101:1007-1008

Thatje S (2015) The Science of Nature - a new era, a new name for Naturwissenschaften. Sci Nat 102:1252. doi:10.1007/s00114014-1255-2 
List of Referees (number of reviews in brackets if more than one)

\author{
Abella J (2), Spain \\ Aerts P (2), Belgium \\ Agnarsson I (3), Puerto Rico \\ Aguirre-Fernandez G, New Zealand \\ Ahlberg PE, Sweden \\ Albert S (3), Germany \\ Albo M, Uruguay \\ Alexander K, UK \\ Allan E, Switzerland \\ Amsalem E, USA \\ Aoyama J, Japan \\ Arenas A, USA \\ Ascoli A, Italy \\ Asis JD, Spain \\ Aspbury A, USA \\ Astua D, Brazil \\ Atkinson C, USA \\ Avargues-Weber A (3), UK \\ Averianov A, Russian Federation \\ Aviles L, Canada \\ Azar D, France \\ Bachand M, Canada \\ Baer C (2), USA \\ Baltzley M (2), USA \\ Barrett P, UK \\ Barrozo RB, Argentina \\ Beaulieu M (2), France \\ Bechsgaard J, Denmark \\ Behmer S, USA \\ Bell P, Australia \\ Bellwood D, Australia \\ Belpaire C, Belgium \\ Beltran S, France \\ Ben N (2), USA \\ Benton M (3), UK \\ Bergman TJ, USA \\ Bergmann J, Chile \\ Bernhagen J, Germany \\ Bianucci G, Italy \\ Biard C, France \\ Bilde T, Sweden \\ Billen J (2), Belgium \\ Billet G, Germany \\ Billeter JC (2), The Netherlands \\ Birkhofer K, Sweden \\ Bize P (2), UK \\ Blackstone N, USA \\ Blamires S, Australia \\ Bockoven A, USA \\ Boeddeker N, Germany \\ Bohm M (2), UK
}

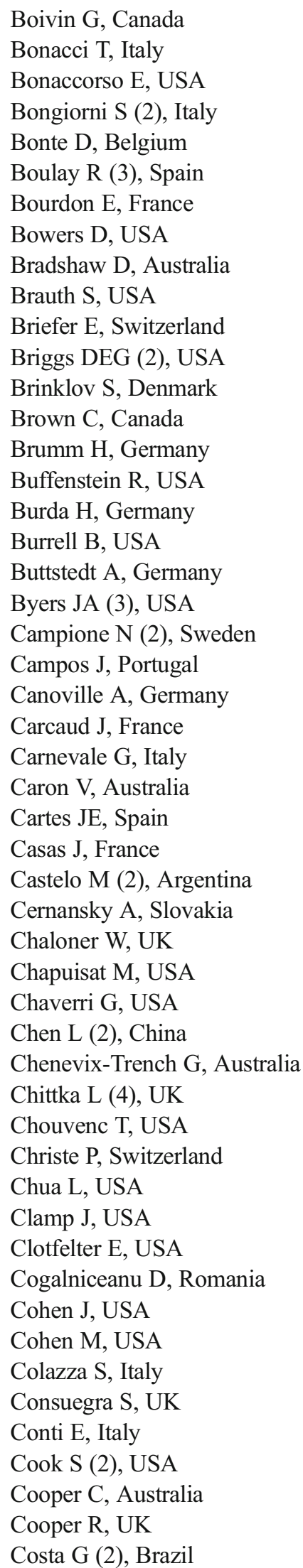

Cox P, UK

Cremer S, Germany

Crewe RM (2), South Africa

Cribb B, Australia

Criscuolo F, France

Croft DA, USA

Cryan P (2), USA

Cucina A, Mexico

Cumming G, South Africa

Dacke M, Sweden

Daniel I, France

David J, France

De Filippis B, Italy

Decombeix AL, France

Dehnhard M, Germany

Dejean A (5), French Guiana

Del Claro K (2), Brazil

Delhey K, Australia

D'Emic M, USA

DeMuizon C, France

Denlinger DL, USA

Desojo JB, Argentina

Detrain C (4), Belgium

Devoto $\mathrm{M}$, Argentina

Dicke M, The Netherlands

Dickman C, Australia

Digweed S (2), Canada

Dijkstra P, UK

Dimitrov D, Bulgaria

Dixson B, Australia

Dodson P (2), USA

Doetterl S, Germany

Domingo E (2), Spain

Donato R, Italy

Donoghue P (2), UK

Dreyer Jamin, USA

Drijfhout F, UK

Duckett J, UK

Duffus A, USA

Duffy S (2), USA

Durica D, USA

Dweck H, Germany

Eberhard W, Costa Rica

Edgar H, USA

Ellis A, South Africa

Eltz T, Germany

Endler J (2), Australia

Erokhin V, Italy

Etges W, USA

Evangelista D, USA

Evans S (2), UK 
Fabrezi M (2), Argentina

Fanzani A, Italy

Feldhaar H, Germany

Feldmann R, USA

Fenton MB, Canada

Ferree E (2), USA

Feunteun E, France

Fiedler K, Austria

Field R, UK

Fitzgerald E (2), Australia

Fleishman L (2), USA

Flower T (2), South Africa

Foissner W, Austria

Fossette S, UK

Francke W, Germany

Franks NR (2), UK

Frederich B, Belgium

Froebisch J, Germany

Frouz J, Czech Republic

Full R, USA

Fulton CJ, Australia

Gaetano L, Argentina

Gasparini J, France

Gatesy J, USA

Gauthier M, France

Geisler J, USA

Germonpre M, Belgium

Gheerbrant E, France

Gimenez L, UK

Giurfa M, France

Glaw F, Germany

Glazier D, USA

Glover B, UK

Gomez-Diaz C, Germany

Gordon D (2), USA

Gorman M, USA

Grebenok R, USA

Gregoric M, Slovenia

Gronenberg W, USA

Groot A, The Netherlands

Grozinger C (2), USA

Guerra R, Brazil

Guo X, China

Guschina I (2), UK

Hagler J, USA

Haldar C, India

Halpern M, Israel

Hameister H, Germany

Han YS, Taiwan

Hanke W, Germany

Hanks L, USA

Hanley D, Canada

Hanula J, USA
Hardus M, The Netherlands

Harland D (2), New Zealand

Harrison J, USA

Harvey J (2), The Netherlands

Hawkins B, USA

Heckert A, USA

Heeb P, France

Hefetz A, Israel

Helantera H, Finland

Hempel de Ibarra N (2), UK

Hepburn R, South Africa

Herberstein ME (2), Australia

Herczeg G, Hungary

Herrel A (2), France

Herzog F (2), Switzerland

Hickey M, Australia

Hill G, USA

Hiltpold I, USA

Hoelldobler B (2), USA

Holmes R, Canada

Holmes M, Australia

Honza M, Czech Republic

Horner J, USA

Hotopp J, USA

Houde P, USA

Humber R, USA

Ianora A, Italy

Ibe B, USA

Ilmoniemi RJ, Finland

Irmis R, USA

Ivens A, USA

Jander R, USA

Jandt J, USA

Janussen D, Germany

Jayne B, USA

Jeschke M, Canada

Jin X, China

Joel DM, Israel

Johnson J, USA

Johnson M, USA

Jones H, UK

Joplin K, USA

Jorgensen C, Norway

Joyce W, Switzerland

Kalthoff D, Sweden

Kanamori A, Japan

Karampelas S, Switzerland

Kavaliers M, Canada

Kear B, Sweden

Keating J, UK

Keil T (2), Germany

Kellermann V, Australia

Kelly D, New Zealand
Kerp H, Germany

Khannoon E, Egypt

Kiel S, Germany

Kilgas P (2), South Africa

Kindsvater H, Canada

Kirejtshuk A, Russian Federation

Kleineidam C, Germany

Klein N, Germany

Klinbunga S, Thailand

Klompen H, USA

Kluen E, Finland

Koch H, USA

Kodandaramaiah U (2), India

Koertner G, Australia

Kogan I, Germany

Kokuer M, UK

Kovacs-Hostyanszki A (2), Hungary

Kraus FB, Germany

Kristan WB Jr., USA

Kriwet J, Austria

Kuijper B (2), UK

Kuntner M (3), Slovenia

Lach L, Australia

Lambdin P (2), USA

Lambert $\mathrm{O}$, Belgium

Langer M, Brazil

Larson E, USA

Lattorff HM, Germany

Laumann R (2), Brazil

Laurin M, France

Lawver D, USA

Lazzari CR, France

Leal WS, USA

Lee Chang KJ (2), Australia

Lemos FJ, Brazil

Lenz P, USA

Leonhardt S (3), Germany

Letnic M, Australia

Levit G (2), Canada

Li J (2), China

Liao J, USA

Liebig J, USA

Lihoreau M, France

Liu Z, China

Locatelli F, Argentina

Lockley MG, USA

Loefstedt C, Sweden

Lohmann K (2), USA

Lorenzi MC, Italy

Lou Y, China

Lukacs J, USA

LunauK (6), Germany

Luo ZX (2), USA 
Luschi P, Italy

Lyytinen A, Finland

Makovicky P, USA

Malcicka M, The Netherlands

Malka O, Israel

Mandahl N, Sweden

Mangan B (2), USA

Mankin R (2), USA

Maoka T (2), Japan

Marivaux L, France

Martin L, USA

Martinez J, Chile

Martinez JG, Spain

Masters J, South Africa

Matzke N, USA

McCairns S, Finland

McCarthy T, Ireland

McIntosh M (2), Germany

McRae S, USA

Meachen J, USA

Measey GJ, South Africa

Medel R, Chile

Menzel F (2), Germany

Merila J (2), Finland

Meseguer J, Spain

Meyer-Berthaud B, France

Meyer-Rochow V (2), Germany

Michel K, USA

Miller M, Japan

Miserez A, Singapore

Mitchell K, South Africa

Mitchell J, USA

Mohan SV, India

Moller A (2), France

Mongeau JM, USA

Monnin T, France

Monteiro F, Portugal

Mora EC, Cuba

Morehouse N (2), USA

Morellet N, France

Moskat C (2), Hungary

Mota P, Portugal

Moya A, USA

Mueller J, Germany

Muheim R, Sweden

Munoz-Garcia A (2), USA

Myers C, USA

Nanda I, Germany

Narazaki T, Japan

Naug D, USA

Nehring V, Germany

Nel A, France

Nelson G, USA
Nesbitt S, USA

Nespolo R, Chile

Newton I, USA

Nieh J, USA

Niven RK, Australia

Numata H (2), Japan

Nunes T, Brazil

Nunez P, USA

O’Connor C (2), Canada

Ohira T, Japan

Oldroyd BP (3), Australia

Oliphant A, UK

Oliver PG, UK

Olsson M, Australia

Opell B, USA

Osiejuk T, Poland

Osorio D, UK

Ossenkopp KP, Canada

Parnell L (2), USA

Pavoine S, France

Peer BD (2), USA

Pekar S, Czech Republic

Pelabon C, Norway

Penna M, USA

Peretti A, Argentina

Perez Igualada L, Spain

Perez-Mellado V, USA

Perkovsky EE, Ukraine

Perry J, USA

Peterson AT, USA

Petit E, France

Petschenka GP, USA

Phillips J, USA

Pinno B, Canada

Pirk C (2), South Africa

Pisanski K, Canada

Pisera A, Poland

Pizo Ferreira M, Brazil

Poethke H, Germany

PoinarJr G, USA

Polly D, USA

Popham H, USA

Poulsen M, Denmark

Prato F (2), Canada

Presley S, USA

Prestianni C (2), Belgium

Prevosti F, Argentina

Pringle E, USA

Proctor H, Canada

Pruett-Jones S, USA

Purnell M, UK

Pyenson N, USA

Radwan A, Egypt
Rahman I, UK

Raine N, Canada

Rangel J, USA

Real R, Spain

Reddon A, Canada

Reinke B, USA

Renault D, France

Reydon T (2), Germany

Reynolds A, UK

Richardson PJ, Canada

Riegert J, Czech Republic

Riek A (2), Germany

Ringseis R, Germany

Rivera Gutierrez HF, Belgium

Rivers D, USA

Robert D, UK

Rocchini D, Italy

Rodrigues A, Brazil

Rodriguez R, USA

Roemer H (2), Austria

Roessler W, Germany

Rogell B (2), Sweden

Rogers L, USA

Rohde M, Germany

Rojas Leon JC, Mexico

Rosengaus RB, USA

Roth Z, Israel

Rothschild B, USA

Rougier G, USA

Rozylowicz L, Romania

Ruiz-Gonzalez M, Spain

Sacchi R, Italy

Saccone P, Finland

Samuels J, USA

Sanciangco J, USA

Scharf I, Israel

Schirhagl R, The Netherlands

Schleyer M, Germany

Schlyter F, Sweden

Schmidt J, USA

Schmitz H (2), Germany

Schneider J, Germany

Schneider S, USA

Schou M, Denmark

Schroeder J, Germany

Schultz T, USA

Schultze HP (2), USA

Scofield P, New Zealand

Scordato E, USA

Seeley T (3), USA

Seid M, USA

Selden P (2), USA

Seoane Pinilla J, Spain 
Sharma PP, USA

Sherbrooke W, USA

Shik J, USA

Shine R, Australia

Siede R, Germany

Sim CK, Singapore

Smith J, USA

Smith T, Belgium

Sorensen JG, Denmark

Standen E, Canada

Stapley J, UK

Stavenga D (3), The Netherlands

Stawski C, Australia

Steiger S, Germany

Steel L, UK

Stelinski L, USA

Stouthamer R, USA

Strassmann JE, USA

Strohm E, Germany

Sutton G, UK

Svanback R, Sweden

Svensson G, Sweden

Svensson A, Sweden

Swallow J, USA

Szyszka P (2), Germany

Tabata J (2), Japan

Tatarenkov A (2), USA

Tautz J, Germany

Taylor R, UK

Tebayashi S, Japan

Telschow A, Germany

Temple-Smith P (2), Australia

Therrien F, Canada

Thomas B, UK
Tilgar V (2), Estonia

Tintori A, Italy

Tobalske B, USA

Tomescu M (2), USA

Tomioka K (2), Japan

Torday J, USA

Torto B, Kenya

Tragust S, Germany

Traver B, USA

Tricarico E, Italy

Trinajstic KM, Australia

Trosko J, USA

Tseng ZJ, USA

Tsubaki Y, Japan

Tsuda A, Japan

Tsutsui N, USA

Tullberg B, Sweden

Tumlinson J, USA

Turbill C, Australia

Turlings T, Switzerland

Tybur J, The Netherlands

Tzeng WN, Taiwan, Republic of China

Uhen M, USA

Uhl G, Germany

Upchurch P, UK

Valdes A, France

Van Damme R, Belgium

Van den Driesche S, UK

VandenBrooks JM, USA

Van Duijn C, The Netherlands

Vesely P, Czech Republic

Victorsson J (2), Sweden

Vollmer G, Germany

Vrsansky P, Slovakia
Vuts J, UK

Waite H, USA

Wall CE, USA

Walton V, USA

Wang Q, China

Ware J, USA

Warnecke L (3), Germany

Wasielesky Jr. W, Brazil

Watanabe H (2), Japan

Waterworth R (2), USA

Wcislo W (2), Panama

Wells H (2), USA

Werdelin L, USA

Westwood J, USA

Whiting M, Australia

Wielgat R, Poland

Wiens G, USA

Wiklund C, Sweden

Wilder S, Australia

Williams L, France

Wilson J, USA

Wiltschko R (2), Germany

Wirsing A, USA

Wisenden B (2), USA

Witte V, Germany

Wojciechowski M (2), Poland

Wolff J, Germany

Wood J, New Zealand

Woodburne M, USA

Worthy T, Australia

Zalucki M, Australia

Zara FJ, Brazil

Zhou Z, China

Zhu M, China 